This item was submitted to Loughborough's Research Repository by the author.

Items in Figshare are protected by copyright, with all rights reserved, unless otherwise indicated.

\title{
Solitary waves in a model of dendritic cable with active spines
}

PLEASE CITE THE PUBLISHED VERSION

LICENCE

CC BY-NC-ND 4.0

\section{REPOSITORY RECORD}

Coombes, S., and P.C. Bressloff. 2019. "Solitary Waves in a Model of Dendritic Cable with Active Spines". figshare. https://hdl.handle.net/2134/841. 


\title{
SOLITARY WAVES IN A MODEL OF DENDRITIC CABLE WITH ACTIVE SPINES
}

\author{
S COOMBES* AND P C BRESSLOFF ${ }^{\dagger}$
}

\begin{abstract}
.
We consider a continuum model of dendritic spines with active membrane dynamics uniformly distributed along a passive dendritic cable. By considering a systematic reduction of the HodgkinHuxley dynamics that is valid on all but very short time-scales we derive 2 dimensional and 1 dimensional systems for excitable tissue, both of which may be used to model the active processes in spine-heads. In the first case the coupling of the spine head dynamics to a passive dendritic cable via a direct electrical connection yields a model that may be regarded as a simplification of the Baer and Rinzel cable theory of excitable spiny nerve tissue [3]. This model is computationally simple with few free parameters. Importantly, as in the full model, numerical simulation illustrates the possibility of a traveling wave. We present a systematic numerical investigation of the speed and stability of the wave as a function of physiologically important parameters. A further reduction of this model suggests that active spine-head dynamics may be modeled by an all or none type process which we take to be of the integrate-and-fire (IF) type. The model is analytically tractable allowing the explicit construction of the shape of traveling waves as well as the calculation of wave speed as a function of system parameters. In general a slow and fast wave are found to co-exist. The behavior of the fast wave is found to closely reproduce the behavior of the wave seen in simulations of the more detailed model. Importantly a linear stability theory is presented showing that it is the faster of the two solutions that is stable. Beyond a critical value the speed of the stable wave is found to decrease as a function of spine density. Moreover, the speed of this wave is found to decrease as a function of the strength of the electrical resistor coupling the spine-head and the cable, such that beyond some critical value there is propagation failure. Finally we discuss the importance of a model of passive electrical cable coupled to a system of integrate-and-fire units for physiological studies of branching dendritic tissue with active spines.
\end{abstract}

Key words. cable equation, dendritic spines, integrate-and-fire

AMS subject classifications. 92C20,34A34,76B25

1. Introduction. Many of the classes of neurons that are distinguished in neurophysiology have large branching structures typically referred to as dendritic trees. The majority of synapses, both excitatory and inhibitory, terminate on dendrites, so it has long been assumed that dendrites somehow integrate inputs to produce neuronal output. Importantly over $90 \%$ of all excitatory synapses that occur in the cortex are located on so-called dendritic spines [20]. Spines are tiny specialized protoplasmic protuberances that cover the surface of many types of neurons. They may be regarded as knob-like appendages consisting of a bulbous spine-head and a spine-stem. They typically occupy $20-70 \%$ of the total dendritic membrane [36]. Since the input impedance of a spine-head is typically large a small excitatory conductance, arising from the activation of a synapse say, can produce a large local depolarization. Moreover, the thin spine-stem neck provides an axial resistance that partially decouples the spine-head dynamics from the dendritic tree. Hence, when equipped with excitable membrane the spine-head provides a favorable site for the initiation of an action potential.

Much of the theoretical work in modeling the computational properties of dendritic structures has focused on the passive properties of dendritic tissue where the techniques of cable theory and compartmental modeling (see for example [19])) may

*Nonlinear and Complex Systems Group, Department of Mathematical Sciences, Loughborough University, Leicestershire, LE11 3TU, UK. S.Coombes@Lboro.ac.uk

${ }^{\dagger}$ Nonlinear and Complex Systems Group, Department of Mathematical Sciences, Loughborough University, Leicestershire, LE11 3TU, UK. P.C.Bressloff@Lboro.ac.uk 
be brought to bear. With these approaches it has been possible to elucidate the role of the dendritic tree in processing input at synapses spatially distributed throughout its structure (see [8] for a review). However, it has long been theorized that the dendritic tree can behave as more than just some complex spatio-temporal filter for synaptic inputs. In fact there exist several studies of passive dendritic models with active membrane sites, either directly on the dendritic membrane [4] or placed in the spine-head $[27,37,32,29,35]$. Experimental data suggests that distal dendrites (at least in CA1 pyramidal cells) are endowed with a variety of excitable channels, including $\mathrm{Ca}^{2+}$ channels in the spine-head, that can support an all-or-nothing response to an excitatory synaptic input. For a historical perspective on the controversy surrounding the generation of action potentials and travelling waves in dendrites we refer the reader to the review by Segev and Rall [36]. It is only relatively recently that experimental observations have confirmed the generation of action potentials in the dendrites. Indeed new experimental techniques have highlighted the nonlinear nature of signal processing in both dendrites and dendritic spines. Hence, theoretical explorations of such biophysical nonlinearities may have important consequences for our understanding of neural processing. Since the biophysical properties of spines can be modified by experience in response to patterns of chemical and electrical activity, morphological and electro-chemical changes in populations of dendritic spines are thought to provide a basic mechanism for learning in the nervous system. Moreover, spine geometry can provide diffusive resistance so that a spine with voltage dependent $\mathrm{Ca}^{2+}$ channels can accumulate $\mathrm{Ca}^{2+}$ with repeated firing. This may form the basis of synaptic plasticity via a second messenger system. In the language of artificial neural networks spinespine interactions have been suggested as a basis for realizing so-called higher order or Sigma-Pi networks [30]. In fact the properties of spines have been linked with i) classes of Hebbian synaptic learning rules capable of learning temporal associations [1] ii) the implementation of logical computations [37] iii) coincidence detection [23] iv) orientation tuning in complex cells of visual cortex [26] and v) the amplification of distal synaptic input inputs [27] (see [20] for an overview of these results). The last point is addressed in this paper.

It has been suggested by Shepherd et al. [38] that if the heads of dendritic spines have excitable membrane properties, the spread of current from one spine along the dendrites could bring adjacent spines to their thresholds for impulse generation. The result would be a sequence of spine-head action potentials, representing a saltatory propagating wave in the distal dendritic branches. This hypothesis is born out in the analysis of Baer and Rinzel for a passive uniform (possibly branching) dendritic tree coupled to a population of excitable dendritic spines [3]. In this continuum model the active spine-head dynamics is modeled with Hodgkin-Huxley kinetics whilst the (distal) dendritic tissue is modeled with the cable equation. The spine-head is coupled to the cable via a spine-stem resistance that is modeled as a simple ohmic resistor that delivers a current proportional to the number of spines at the contact point. The geometry of the spine-head is ignored and spines are considered isopotential. There is no direct coupling between neighboring spines; voltage spread along the cable is the only way for spines to interact. In a numerical study of their model Baer and Rinzel found that a brief synaptic activation of one or more spines could indeed lead to action potential generation and a subsequent chain reaction type propagation for a wide range of spine densities. For a given number of spines, chain reactions depend crucially on their spatial distribution; low uniform density of spines, or dense clusters which are too far apart may preclude signal propagation. Moreover, too large a choice 
for the spine stem resistance may lead to propagation failure [3]. A more analytically tractable version of the Baer and Rinzel model has been presented by Zhou [40]. In this model the 4 dimensional Hodgkin-Huxley kinetics in the spine-head is replaced by a 1 dimensional Nagumo type dynamics. This essentially treats the spine-head as a nonlinear membrane such that the spine-head voltage has two stable fixed points and one unstable fixed point. Traveling fronts are equivalent to heteroclinic connections between the two stable fixed points. For small spine-stem resistance it may be shown that a unique traveling front exists and that the speed of the front is larger for a smaller spine density. The lack of a recovery variable in the model of Zhou means that traveling pulses of the type observed by Baer and Rinzel are not possible. However, numerical simulations with the inclusion of a recovery variable (FitzHugh-Nagumo dynamics) show that the speed of a traveling pulse is close to that of a traveling front in the model without recovery. A study of the steady state solutions in models of this type (with Nagumo dynamics and no recovery variable) may be found in [10].

In this paper we consider a mathematical reduction of the original Baer and Rinzel model that allows one to analyze both the existence and stability of traveling pulses. In the original Baer and Rinzel model the active membrane in the spine-head is described with the Hodgkin-Huxley equations. The reductive process we utilize leads naturally to a model where the active processes in the spine-head are described by a two dimensional dynamical system reminiscent of the FitzHugh-Nagumo model. An advantage of utilizing a well defined reductive process, in contrast to simply invoking a simpler FitzHugh-Nagumo dynamics, is that all parameters in the model have a direct physiological interpretation. This so-called reduced Baer and Rinzel model is also attractive from a computational perspective since it allows large scale simulations to be performed with relative ease. We then use the fact that events in the spinehead are typically of the all-or-nothing variety to motivate a further reduction of the model. We regard the spine-head dynamics as one that generates pulse-shapes at times defined by some thresholding process. These pulses are instantaneously communicated to the cable via the spine-stem resistance, whilst the dynamics in the spine-head is driven by the flow of current from the cable to the spine-head via the spine-stem. In effect we introduce an integrate-and-fire type mechanism for the spine-head dynamics via a systematic reduction of the Hodgkin-Huxley equations. Integrate-and-fire (IF) dynamics have been studied by many authors since they are analytically tractable and submit to a form of linear stability analysis (see [9] for a review). In section 2 we introduce the mathematical model of Baer and Rinzel and discuss the reduction of the excitable spine-head membrane dynamics. Numerical simulations are presented to demonstrate the possibility of traveling pulses. The speed of this wave is numerically calculated as a function of system parameters and results are shown to be consistent with those found in the full model. In section 3 we introduce the analytically tractable IF model and show how one may explicitly construct the profile of traveling wave solutions. We then go on to show how one may self-consistently determine the speed of such traveling waves as a function of system parameters and the shape of the pulse generated in the spine-head. Direct numerical simulations of the IF model are shown to agree with our analysis. In section 4 we develop a linear stability analysis of the map of firing times of the IF process that can be used to determine the stability of traveling waves. Of the two possible wave speeds that are found to exist, for a given set of system parameters, we are able to show that it is always the faster of the two that is stable. In summary the reduced IF version of the Baer and Rinzel model supports stable traveling pulses with speeds that decrease as a function of (constant) spine 
density and spine-stem resistance. As in the full model propagation failure results for insufficient spine density or too great a spine-stem resistance. Moreover, using realistic values for parameters in the IF model (obtained from the reductive process) we obtain wave speeds in agreement with those found in the more detailed model of Baer and Rinzel. Finally in section 5 we compare the response of a passive cable studded with passive spines for synaptic inputs directly to the spines and directly to the dendritic shaft. In the conclusion we argue that not only does the IF model of spiny dendritic cable provide a mathematically tractable perspective from which to understand the results of Baer and Rinzel, but that it also may serve as a basis for testing the computational role of active membrane in spine-heads.

2. A biophysical model. In order to analyze the interactions between spines Baer and Rinzel formulated a new type of cable theory in which the distribution of spines is treated as a continuum. The formulation retains the notion that there is no direct electrical coupling between neighboring spines. Voltage spread along a uniform passive cable is the only way for the spines to interact; spines are electrically independent from one another. The dynamics of action potential generation at the neural cell body or soma is perhaps most accurately described with Hodgkin-Huxley dynamics. It is common practice to use this dynamics as a model of active membrane in the dendritic tree. However, it is worth noting that there are in fact differences at the biophysical level between active membrane in the soma and the dendrites [14]. Also, antidromic (soma to dendrites) action potentials can propagate well into the dendritic tree, as well as along the outgoing axon [11]. Indeed the geometry of a typical dendritic tree means that it is possible for $\mathrm{Na}^{+}$mediated action potentials to travel from the soma and proximal dendrites towards the thinner distal ones but not vice versa. Since the threshold for initiation of an action potential in distal arbors and spines depends crucially on the spatial distribution of the excitatory input the dendritic tree appears capable of solving realistic pattern discrimination tasks [25]. In the Baer and Rinzel model the active membrane in the spine-head is modeled by Hodgkin-Huxley kinetics. The continuum model of Baer and Rinzel, with spine density per unit area of dendritic membrane at location $x$ given by $\rho(x)$, is expressed by the following equations:

$$
\begin{aligned}
& C \frac{\partial V}{\partial t}=-g_{L}\left(V-V_{L}\right)+\frac{1}{r_{a} \pi d} \frac{\partial^{2} V}{\partial x^{2}}+\rho(x) \frac{\widehat{V}-V}{r} \\
& \widehat{C} \frac{\partial \widehat{V}}{\partial t}=-I(\widehat{V}, m, n, h)-\frac{\widehat{V}-V}{r}
\end{aligned}
$$

Equation (2.1) describes the dynamics of an infinite uniform passive cable of diameter $d$ with voltage $V(x, t)$ such that the last term on the right hand side is proportional to the flow of current between the cable shaft and the spine-head above the shaft via the ohmic spine-stem resistance of strength $r$. The terms $V_{L}$ and $g_{L}$ are respectively a constant leakage reversal potential and a leakage conductance per unit area of membrane. The parameter $r_{a}$ represents the intracellular resistance per unit length of the cable. The electronic length constant $\lambda$ is given by $\lambda^{2}=1 /\left(\pi d r_{a} g_{L}\right)$ and the membrane time constant (of the dendritic cable) by $\tau=C / g_{L}$. For the remainder of this paper we consider the case that the spine density function is a constant with $\rho(x)=\rho$ for all $x$. The excitable dynamics of the voltage in the spine-head $\widehat{V}(x, t)$ is driven by the flow of current from the shaft to the spine and is described with equation (2.2). From now on we choose specific membrane capacitances $C$ and $\widehat{C}$ such that $C=$ 
$\widehat{C}$ and take $C=1$ without loss of generality. It is also convenient to choose a length scale such that $r_{a} \pi d=1$. In the Hodgkin-Huxley model of excitable nerve tissue the membrane current arises mainly through the conduction of sodium and potassium ions through voltage dependent channels in the membrane. The contribution from other ionic currents is assumed to obey Ohm's law. In fact the Hodgkin-Huxley dynamics, described with the use of the function $I(\widehat{V}, m, n, h)$, is considered to be a function of $\widehat{V}$ and three time and voltage dependent conductance variables $m, n$ and $h$ :

$$
I(\widehat{V}, m, n, h)=g_{K} n^{4}\left(\widehat{V}-V_{K}\right)+g_{N a} h m^{3}\left(\widehat{V}-V_{N a}\right)+g_{L}\left(\widehat{V}-V_{L}\right)
$$

where $g_{K}, g_{N a}$ and $g_{L}$ are constants and $V_{L}, V_{K}$ and $V_{N a}$ represent the constant membrane reversal potentials associated with the leakage, potassium and sodium channels respectively. For simplicity we have considered the currents due to the leakage terms in the model of the cable and those in the model of the spine-head to be identical. The conductance variables $m, n$ and $h$ take values between 0 and 1 and approach the asymptotic values $m_{\infty}(\widehat{V}), n_{\infty}(\widehat{V})$ and $h_{\infty}(\widehat{V})$ with time constants $\tau_{m}(\widehat{V}), \tau_{n}(\widehat{V})$ and $\tau_{h}(\widehat{V})$ respectively. Summarizing, we have that

$$
\tau_{X}(\widehat{V}) \frac{\mathrm{d} X}{\mathrm{~d} t}=X_{\infty}(\widehat{V})-X, \quad X \in\{m, n, h\}
$$

The six functions $\tau_{X}(\widehat{V})$ and $X_{\infty}(\widehat{V}), X \in\{m, n, h\}$, are obtained from fits with experimental data. The details of the final Hodgkin-Huxley description of nerve tissue are completed in appendix A. Baer and Rinzel have shown that the combination of diffusion along the cable combined with input from electronically separated active spines can lead to the propagation of a traveling wave. In essence a brief external input, say in the form of a synaptic current, leads to a spread of potential along the cable. The dynamics in the spine head is driven by this potential and for sufficiently large drive an action potential may be generated. There is then a large re-injection of current back into the dendrite that causes a further spread of potential along the cable so that the process is self-perpetuating. The natural refractoriness of the HodgkinHuxley dynamics means that spines in the wake of the wave are not re-excited. Thus one expects firing activity in the spines to ride the crests of a succession of diffusing pulses in the dendritic cable. Simulations show that the speed of this traveling wave decreases as a function of the spine density [3, 40]. Moreover there is propagation failure for too large a spine-stem resistance or too small a spine density. For $0<r \ll 1$ the potential difference between the spine head and the dendrite becomes negligible. In this regime the system behaves like an excitable cable (much like an axon) and supports travelling waves with constant profiles [3, 40]. For sufficiently large $r$ the system de-couples into a passive cable and a set of electrically isolated (excitable) spine-heads and there are no travelling wave solutions.

2.1. A reduction of the Baer and Rinzel model. Although the inclusion of the three gating variables in the Hodgkin-Huxley dynamics yields a realistic model of excitable tissue it does lead to an element of mathematical intractability. Ideally for the purposes of analysis one would like a reduction of the 4 dimensional Hodgkin-Huxley equations that maintains the essentials of a spiking neuron model. A systematic method for achieving such a reduction has been proposed by Abbott and Kepler [2] and is developed in [17]. Here we use this technique of equivalent potentials to obtain a reduced version of the original 5 dimensional Baer and Rinzel model to one that is only 3 dimensional. Numerics are used to show that this, computationally 
simpler, model can capture the essential features of the full model, namely traveling pulses with quantitatively similar propagation speeds. In the next section we extend this reduction process to motivate the introduction of a new IF version of the Baer and Rinzel model that is analytically tractable.

The reduction process starts with the conductance based Hodgkin-Huxley model which depends upon 4 dynamical variables. A space clamped portion of excitable membrane is described by

$$
\frac{\mathrm{d} \widehat{V}}{\mathrm{~d} t}=-I(\widehat{V}, m, n, h)+J
$$

where $J$ represents any injected currents. It is well known that the time-scale associated with changes in $m$ and $\tau_{m}$ is much smaller than those associated with $h$ and $n$. Thus $m$ will reach its asymptotic value quickly, motivating the replacement of $m$ by its asymptotic value $m_{\infty}(\widehat{V})$. This instantaneous approximation reduces the number of dynamic variables from 4 to 3 at the expense of accuracy over very short time-scales. A similar reduction for $n$ and $h$ is not appropriate since this would destroy the ability of the model to generate action potentials. Interestingly, on a periodic limit cycle, one observes that $n$ and $h$ are related by an approximately linear relationship. This suggests that $h$ may be eliminated by the replacement $h=a n+b$ for suitable choices of the constants $a$ and $b$. (For the parameters used in the appendix with $J=10$ a numerical least squares fit gives $a=-0.83$ and $b=0.82)$. This approach provides a straightforward method for reducing the dimensionality of the Hodgkin-Huxley system without much effort and has been used by several authors (see for example [34]). Rather than pursue this simple reduction we continue with the introduction of two auxiliary potentials $U_{n}$ and $U_{h}$. The basic idea is to mimic the slower approach of $n$ and $h$ to their asymptotic values by the replacement

$$
X=X_{\infty}\left(U_{X}\right), \quad X \in\{n, h\}
$$

This equation can always be solved exactly for $U_{X}$ since the functions $X_{\infty}$ are monotonic and hence invertible. In practice it is found that the auxiliary potentials $U_{h}$ and $U_{n}$ are remarkably similar. A further reduction is now possible with the replacement of $U_{X}$ by an average over the two potentials. In fact it is simpler still to assume that they are identical and let $U_{h}=U_{n}=U$. Hence, the reduced model is now 2 dimensional with a membrane current $f(\widehat{V}, U)=I\left(V, m_{\infty}(\widehat{V}), n_{\infty}(U), h_{\infty}(U)\right)$. By demanding that the time-dependence of $f$ in the reduced model mimic the time-dependence of $I$ in the full model at constant $\widehat{V}$ it is possible to show that

$$
\begin{aligned}
\frac{\mathrm{d} \widehat{V}}{\mathrm{~d} t} & =-f(\widehat{V}, U)+J \\
\frac{\mathrm{d} U}{\mathrm{~d} t} & =\frac{\mathcal{A}(\widehat{V}, U)}{\mathcal{B}(\widehat{V}, U)} \equiv g(\widehat{V}, U)
\end{aligned}
$$

where

$$
\begin{aligned}
& \mathcal{A}(\widehat{V}, U)=\frac{\partial I}{\partial h}\left[\frac{h_{\infty}(\widehat{V})-h_{\infty}(U)}{\tau_{h}(\widehat{V})}\right]+\frac{\partial I}{\partial n}\left[\frac{n_{\infty}(\widehat{V})-n_{\infty}(U)}{\tau_{n}(\widehat{V})}\right] \\
& \mathcal{B}(\widehat{V}, U)=\frac{\partial f}{\partial h_{\infty}} \frac{\mathrm{d} h_{\infty}(U)}{\mathrm{d} U}+\frac{\partial f}{\partial n_{\infty}} \frac{\mathrm{d} n_{\infty}(U)}{\mathrm{d} U}
\end{aligned}
$$


and $\partial I / \partial h$ and $\partial I / \partial n$ are evaluated at $h=h_{\infty}(U)$ and $n=n_{\infty}(U) . \widehat{V}$ describes the capacitive nature of the cell and $U$ describes the time-dependence of the membrane conductance. In fact $U$ may be regarded as a variable responsible for the refractory period of a neuron. The reduction to a 2 dimensional system allows a direct visualization of the dynamics by plotting the flow in the $(\widehat{V}, U)$ plane. A plot of the nullclines (see the top of figure 2.1) allows the visualization of both the fixed point and the flow (see the bottom of figure 2.1). Standard values for system parameters have been used and may be found in appendix A. In the reduced model $g(\widehat{V}, \widehat{V})=0$ and the nullcline for $\mathrm{d} U / \mathrm{d} t$ is simply the straight line $U=\widehat{V}$. The fixed point for zero external input is found at $(\widehat{V}, U)=(-65,-65)$. Moreover, in this instance the fixed point is stable and the neuron is said to be excitable. When a positive external current is applied the low-voltage portion of the $\mathrm{d} \widehat{V} / \mathrm{d} t$ nullcline may move up until the intersection of the two nullclines falls within the portion of the $\mathrm{d} \widehat{V} / \mathrm{d} t=0$ nullcline with positive slope (see the top of figure 2.1). In this case the fixed point is unstable and the system may support a limit cycle as shown in the bottom of figure 2.1. The system is said to be oscillatory as it may produce a train of action potentials (see the inset for $\widehat{V}$ in the bottom of figure 2.1). Action potentials may also be induced in the absence of an external current for synaptic stimuli of sufficient strength and duration. This reduced model of neural membrane is reminiscent of the FitzHugh-Nagumo dynamics, but with some important differences. For example, the amplitude of action potentials in both the full Hodgkin-Huxley model and the reduced model decrease at high firing rates. In contrast in the FitzHugh-Nagumo model the amplitude is relatively independent of the firing frequency. More importantly though, the reduction process allows one to maintain contact with biological reality so that parameters in the reduced system have physiological interpretation.

We now illustrate the possibility of traveling waves in the reduced Baer and Rinzel model with the aid of a simple compartmental model and numerical simulation. Moreover, for a finite (yet long) piece of cable and realistic choices of parameter values we numerically obtain the speed of a traveling pulse as a function of the spine density. For a cable of length $L$ we discretize space such that the cable is represented by $N$ compartments (with killed end boundary conditions $V(0)=V(L)=0$ ) each of length $\Delta s=L / N$. We then iterate the discretized version of the dynamical system defined by $(2.1),(2.7)$ and $(2.8)$ with a simple Euler method of step size $\Delta t=(\Delta s)^{2} / 4$. Traveling waves are easily initiated in the numerical model by driving the system with a constant input into the spine-head compartment at $x=L$ for a time sufficiently long enough to initiate a firing event. An example of a traveling wave is given in figure 2.2, whilst figure 2.3 shows that the speed of such solitary pulses decreases as a function of the spine density. As in the full model propagation failure occurs for too small a spine density or too large a spine-stem resistance. Apart from increasing $L$ and considering more compartments, an improvement in the accuracy of our numerical results can be obtained by decreasing the choice of step size from $\Delta t=(\Delta s)^{2} / 4$ to $\Delta t=(\Delta s)^{2} / k$ with $k>4$. Alternatively one could implement a more sophisticated numerical scheme to take into account the fact that the active processes in the spine head operate on a different time scale to the processes in the dendritic shaft. For a discussion of such techniques (and in particular the Crank-Nicolson scheme) we refer the reader to the review article by Mascagni and Sherman [24]. However, if one is interested only in the behavior of travelling wave solutions it is possible to obtain numerical results to arbitrary accuracy in a different fashion. To construct traveling wave solutions in the reduced Baer and Rinzel model it is natural to assume solutions 

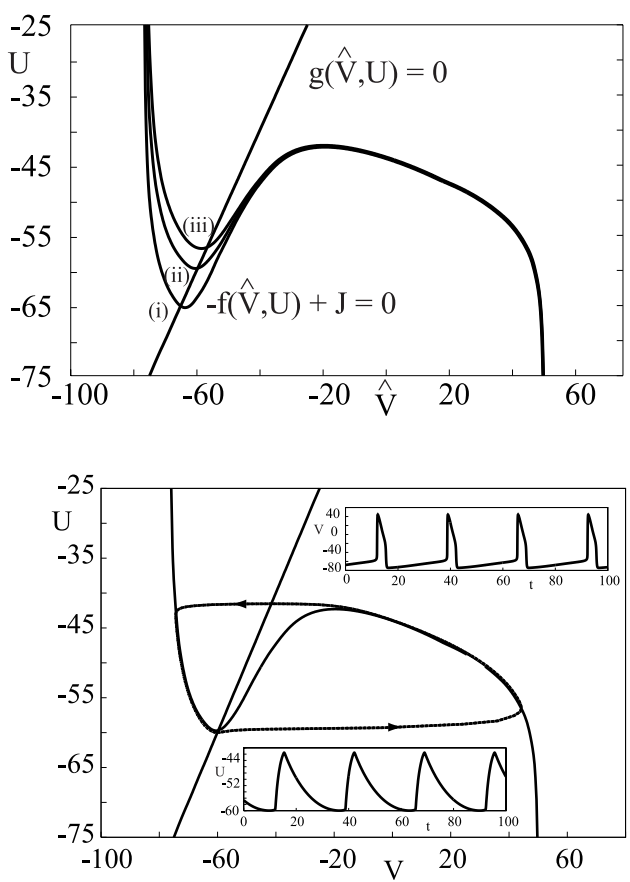

FIG. 2.1. Top: Nullclines of the reduced neuron model with (i) $J=0$, (ii) $J=10$ and (iii) $J=20$. There is a fixed point at $(\hat{V}, U)=(-65,-65)$ with $J=0$. Bottom: The reduced neuron model in an oscillatory regime $(J=10)$ capable of generating a train of spikes (see inset for dynamics of $\hat{V}$ and the refractory variable $U)$.

of the form $V(x, t)=V(\xi)$ where $\xi=c t-x$ and similarly for $\widehat{V}$ and $U$ and to look for homoclinic connections to the resting state. The numerical solution of this problem may be formulated as a two-point boundary value problem parameterized by the speed of the wave [5]. Once an initial homoclinic solution is constructed one may then numerically continue the speed of the wave in one or more system parameters with the aid of a piece of dedicated software such as AUTO97 ${ }^{1}$. Instead we consider a further reduction of the integrate-and-fire model where the voltage in the spine head is considered as a train of pulses generated by a threshold crossing process. As we shall show in the next section this provides an analytically tractable model of dendritic cable with active spines.

3. An integrate-and-fire model. Integrate-and-fire models provide a caricature of the capacitive nature of cell membrane at the expense of a detailed model of the refractory process. To obtain an IF model from the reduced Hodgkin-Huxley model we approximate $U$ by its fixed point value of -65 so that equation (2.7) becomes

$$
\frac{\mathrm{d} \widehat{V}}{\mathrm{~d} t}=-f(\widehat{V},-65)+J
$$

\footnotetext{
${ }^{1}$ available from http://indy.cs.concordia.ca/auto
} 


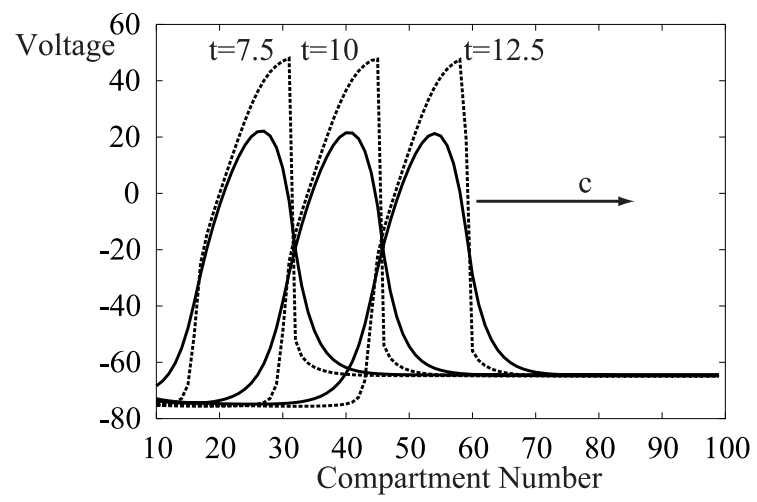

FIG. 2.2. A traveling wave in a compartmental model $(N=100, L=10)$ of the reduced Baer and Rinzel model. $g_{L}=0.3, g_{K}=36, g_{N a}=120, V_{L}=-54.402, V_{K}=-77$ and $V_{N a}=50$, $r=2, \rho=25$. The dashed line shows the voltage in the spine-head, whilst the solid line refers to the voltage in the cable.

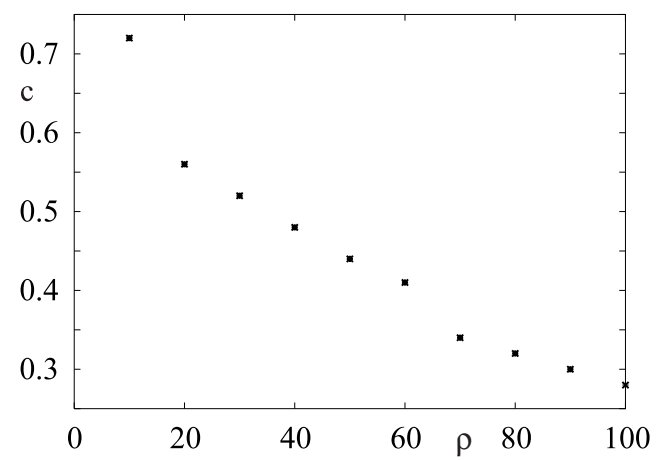

FIG. 2.3. The Speed of a traveling pulse as a function of spine density $\rho$ obtained by numerically integrating the reduced Baer and Rinzel model. Note that the speed of the wave decreases as a function of the spine density and propagation is not possible if the spine density is too low. All parameters as in figure 2.2.

The largest value of $\widehat{V}$ for which $f(\widehat{V},-65)=0$ determines the onset of the spiking regime and can be used to define a threshold $V_{T}$ for firing, since for larger values of $\widehat{V}, \mathrm{~d} \widehat{V} / \mathrm{d} t>0$. It is possible to fit this curve with a polynomial $f(\widehat{V},-65)=$ $a\left(\widehat{V}-V_{A}\right)\left(\widehat{V}-V_{B}\right)\left(\widehat{V}-V_{T}\right)$ where $a=0.008, V_{A}=-65, V_{B}=77.81$ and the threshold for firing is found to be at $V_{T}=-62.56$. A linear model can be obtained by eliminating the dynamics of the variable $U$ in a slightly different fashion. That is, set $U=V$ everywhere so that $g(U, U)=0$ and hence $\mathrm{d} U / \mathrm{d} t=0$. This is a quite severe approximation since $U$ is never very close to $V$ except at the fixed point. A numerical plot of the function $f(\widehat{V}, \widehat{V})$ shows that is approximately linear and can be fit as $f(\widehat{V}, \widehat{V})=a\left(\widehat{V}-V_{T}\right)$ with $a=1.25$ and $V_{T}=-65.65$. If one assumes that it is the spiking part of the waveform generated in the spine-head that affects the shaft dynamics most strongly then it is natural to consider a model of the spinehead that preserves this structure at the expense of an accurate description of the wave-form before and after spiking events. Indeed numerical simulations of the os- 
cillatory Hodgkin-Huxley or reduced Hodgkin-Huxley dynamics support this notion since between spiking events the voltage wave-form is relatively flat (see the inset in the bottom of figure 2.1). Hence, we replace the voltage wave-form generated in the excitable spine-head by a sequence of pulses that are generated whenever the dynamics in the spine-head, driven by current from the shaft, crosses some threshold. The pulse-shape is chosen to mimic that of a real action potential, ie has a biologically realistic magnitude and duration. In more detail we consider a model where

$$
\widehat{V}(x, t)=\sum_{m \in \mathbb{Z}} \eta\left(t-T^{m}(x)\right)
$$

such that $\eta(t)$ specifies the shape of an action potential and the firing times $T^{m}(x)$ are generated by an IF type firing mechanism in the spine-head:

$$
T^{n}(x)=\inf \left\{t \mid U(x, t) \geq h ; t \geq T^{n-1}(x)+\tau_{R}\right\}
$$

For convenience we use the symbol $U$ to denote the state variable describing the IF process in the spine-head. This is not to be confused with the symbol representing the auxiliary potential used in section 2 . One should note that in the IF model we have completely eliminated the slow auxiliary variable (responsible for the shape of an action potential and the relative refractory period) in favor of a simplified description of an action potential as a rectangular pulse. The term $\tau_{R}$ represents an absolute refractory period. It is convenient to introduce such a delay since the IF process cannot mimic the refractory properties of the Hodgkin-Huxley system. For $T^{n}(x)<t<T^{n+1}(x)$ the generator of the IF process, $U(x, t)$, is assumed to evolve according to

$$
\frac{\partial U}{\partial t}=-g_{L}\left(U-V_{L}\right)+\left[\frac{V-U}{r}\right]
$$

subject to reset

$$
\lim _{\delta \rightarrow 0^{+}} U\left(T^{n}(x)-\delta\right)=h, \quad \lim _{\delta \rightarrow 0^{+}} U\left(T^{n}(x)+\delta\right)=g
$$

$h$ is recognized as the threshold for a firing event to occur whilst $g$ represents the reset level of the IF process, with $g<h$. One may adopt a realistic functional form for the waveform $\eta(t)$ using the function approximation techniques outlined in [18] although for the purposes of this section it is merely enough to assume a simple pulse of duration $\tau_{s}$ and height $\eta_{0}$. Note that we have adopted an IF model with a linear decay term rather than the more realistic cubic form described above. This is not too severe an approximation when one bears in mind the success of such models in reproducing experimentally observed spike trains. We choose a realistic value of the threshold as $h=-62.5$ and take the leakage conductance as $g_{L}=1.25$. As in the previous section a numerical simulation demonstrates the possibility of traveling waves (see figure 3.1). In numerical simulations of the reduced model the duration of an action potential is typically around $2 \mathrm{~ms}$ with a peak of about $100 \mathrm{mV}$ above rest. Hence, we take $\tau_{s}=2$ and $\eta_{0}=100$. For more realistic choices of the pulse shape generated in the spine-head one would expect a better agreement with the wave-forms seen in the more detailed models. Importantly however, this much simplified model supports the same type of solitary wave with a correspondingly similar speed to that observed in the full model. Throughout we assume that the refractory period $\tau_{R}$ is sufficiently large so that the 
wake of the traveling wave cannot re-excite any spine-heads that have already fired. Alternatively one may choose the reset level $g$ to be sufficiently negative such that, after reset, the wake cannot drive the system above threshold [13]. The ability of the IF spine-head model to fire repetitively depends upon the choice of the absolute refractory period and the amount of current that leaves the spine-head and enters the dendritic shaft. For realistic choices of the refractory period (of around 2-10 ms) and for a constant input into the spines (IF model) repetitive firing is only observed for large values of the spine-stem resistance where one may regard the spine-head as electrically isolated.

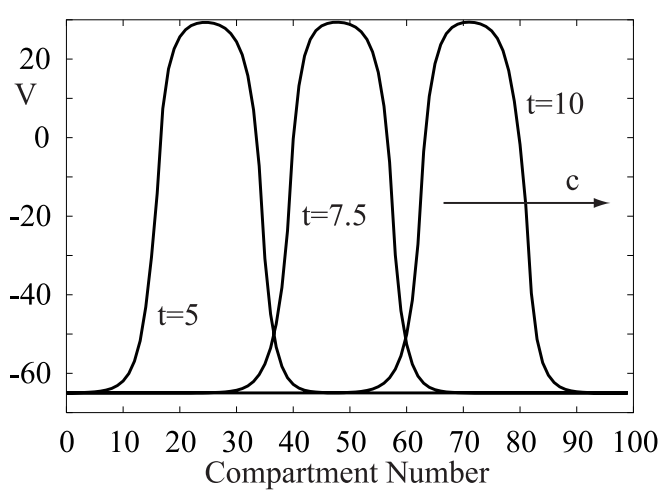

FIG. 3.1. Traveling solitary wave in the IF model of a dendritic cable with active spines obtained by numerically integrating the equations of motion. Parameters are $g_{L}=1.25, V_{L}=-65, h=$ $-62.5, g=-80, r=2, \tau_{s}=2, \eta_{0}=100$ and $\tau_{R}=10 . L=10, N=100$. Note that the speed of the wave is around 1 (in units of electronic length per $\mathrm{ms}$ ).

3.1. Traveling wave solutions. In this section we show how one may explicitly construct the profile of a solitary wave solution in terms of the pulse shape-generated in the spine-head. We define a solitary wave as one that causes the spine-head at $x$ to reach threshold only once at the time $t=T(x) \equiv x / c$. We recognize $c$ as the speed of the wave so that $\widehat{V}(x, t)=\eta(t-x / c)$, which suggests adopting a moving frame co-ordinate system $\xi=c t-x$. The extension to traveling trains instead of pulses is relatively straightforward (see $[6,7])$ and will not be pursued here. As a concrete example we first focus on the case where $\eta(t)$ describes a pulse of height $\eta_{0}$ relative to $V_{L}$ and duration $\tau_{s}$. (In the next section we consider arbitrary pulse shapes). In the traveling co-ordinate frame, and referring all voltages to $V_{L}$ (including the reset and threshold values), we have from (2.1) that

$$
V_{\xi \xi}-c V_{\xi}-\epsilon V=-\frac{\rho}{r} \widehat{V}
$$

where $V_{\xi} \equiv \mathrm{d} V / \mathrm{d} \xi, \epsilon=g_{L}+\rho / r$ and

$$
\widehat{V}(\xi)= \begin{cases}0 & -\infty<\xi<0 \\ \eta_{0} & 0<\xi<c \tau_{s} \\ 0 & \xi>c \tau_{s}\end{cases}
$$


If one is looking for traveling pulses which satisfy $\lim _{\xi \rightarrow \pm \infty} V(\xi)=0$ then the solution to (3.6) takes the form

$$
V(\xi)= \begin{cases}\alpha_{1} \exp \left(m_{+} \xi\right) & -\infty<\xi<0 \\ \alpha_{2} \exp \left(m_{+} \xi\right)+\alpha_{3} \exp \left(m_{-} \xi\right)+\rho \eta_{0} /(\epsilon r) & 0<\xi<c \tau_{s} \\ \alpha_{4} \exp \left(m_{-} \xi\right) & \xi>c \tau_{s}\end{cases}
$$

with

$$
m_{ \pm}=\frac{c \pm \sqrt{c^{2}+4 \epsilon}}{2}
$$

By ensuring the continuity of the solution and its first derivative at $\xi=0$ and $\xi=c \tau_{s}$ one may solve for the unknowns $\alpha_{1} \ldots \alpha_{4}$ as

$$
\begin{aligned}
\alpha_{1} & =\alpha_{3} \frac{m_{-}}{m_{+}}\left[1-\exp \left(-m_{+} c \tau_{s}\right)\right] \\
\alpha_{2} & =-\alpha_{3} \frac{m_{-}}{m_{+}} \exp \left(-m_{+} c \tau_{s}\right) \\
\alpha_{3} & =\frac{\rho \eta_{0}}{\epsilon r} \frac{m_{+}}{\left(m_{-}-m_{+}\right)} \\
\alpha_{4} & =\alpha_{3}\left[1-\exp \left(-m_{-} c \tau_{s}\right)\right]
\end{aligned}
$$

A graphical plot of the exact solution for a traveling pulse is shown in figure 3.2 . As yet the speed of the pulse is undetermined. However, by demanding that the IF

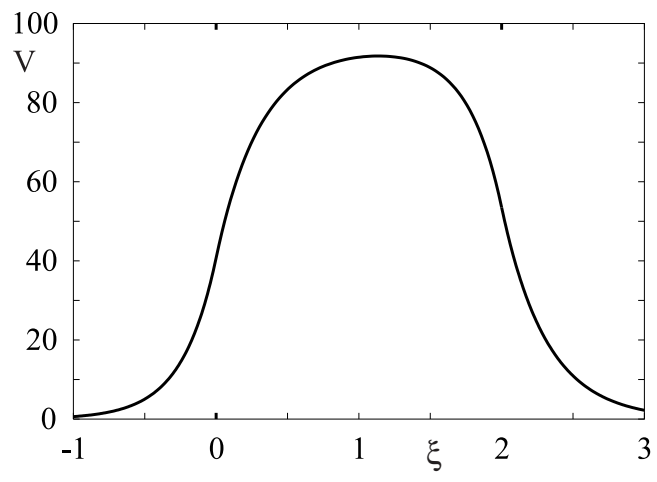

FIG. 3.2. A plot of the exact traveling wave solution in the IF model of a cable with active spines for $c=1, \tau_{s}=2, \rho=25, r=2, g_{L}=1.25$.

process in the spine-head reach threshold at $\xi=0$ and that $\lim _{\xi \rightarrow \pm \infty} U(\xi)=0$ one can determine a self-consistent value for the speed of the traveling wave along the lines outlined by Ermentrout [13] and Bressloff [6, 7]. In the traveling co-ordinate frame we have for the IF process that

$$
c U_{\xi}=-\hat{\epsilon} U+\frac{V}{r}
$$

with $U(0)=h$ and $\hat{\epsilon}=g_{L}+1 / r$. This first order system may be solved as

$$
U(\xi)=\exp (-\xi \hat{\epsilon} / c)\left[h-\frac{1}{c r} \int_{\xi}^{0} V\left(\xi^{\prime}\right) \exp \left(\xi^{\prime} \hat{\epsilon} / c\right) \mathrm{d} \xi^{\prime}\right]
$$


In order for this to be bounded as $\xi \rightarrow-\infty$, the term inside the large parentheses must vanish as $\xi \rightarrow-\infty$. This yields the dispersion relationship for the speed of the pulse as a function of system parameters:

$$
h=\frac{1}{r} \frac{\alpha_{1}}{\left(\hat{\epsilon}+c m_{+}\right)}
$$

A numerical plot of the speed $c$ of a traveling pulse as a function of the spine density

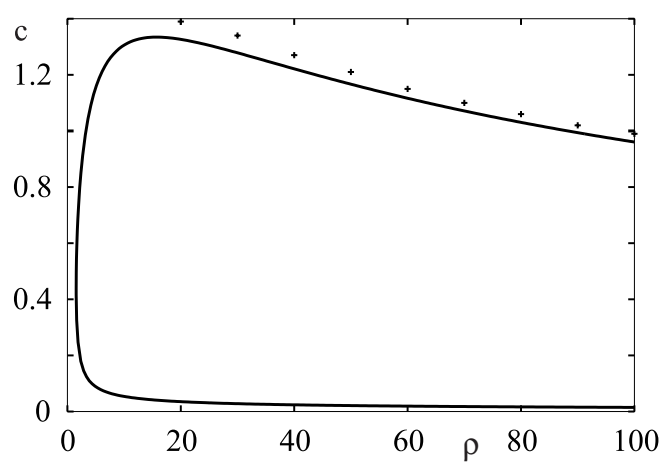

FIG. 3.3. Speed of a traveling pulse as a function of spine density $\rho . g_{L}=1.25, h=2.5, r=2$, $\tau_{s}=2, \eta_{0}=100$. The crosses show the results of a direct numerical simulation of the IF model with $N=200$ and $L=10$ and other parameters as above.

is shown in figure 3.3. Note that there are two solution branches for a given density $\rho$. Direct simulations suggest that it is the upper (faster) branch that is stable. In the next section we establish this result is true at the level of a linear stability analysis. Note that for a wide range of $\rho$ the speed of the stable wave is approximately 1 electronic length per ms, in agreement with the original observations of Baer and Rinzel. Importantly, however, we have an exact expression for the speed of the wave that can easily be solved to obtain the dependence in terms of other system parameters such as the spine stem resistance. Indeed in this model one can find exactly the minimum spine density capable of supporting a traveling pulse as well as extracting information about how the speed decays as a function of spine density. In figure 3.4 we plot the speed of a traveling pulse as a function of the spine-stem-resistance $r$. It is clear, that for realistic choices of the biophysical parameters in the model, that propagation failure can occur for too large a choice of the spine-stem resistance. Moreover, for small $r$, the speed of a stable pulse is very sensitive to $r$, demonstrating that a modifiable value of the spine-stem resistance could have important ramifications for neural processing. Finally in figure 3.5 we show the dependence of the wave speed on the width, $\tau_{s}$, of a rectangular pulse generated in the spine-head. Interestingly, for a fixed height of pulse there is a minimum duration time below which propagation cannot occur. This highlights the fact that it is crucial to model the shape of an action potential in the reduced IF model with biologically realistic choices for the amplitude and duration of the spine-head pulse. For large values of $\tau_{s}$ the speed of the wave approaches a constant value (ie the speed of the wave becomes insensitive to the precise choice of $\tau_{s}$ ).

4. Linear stability of the integrate-and-fire model. For the purposes of linear stability analysis it is more convenient to work in terms of the original variables 


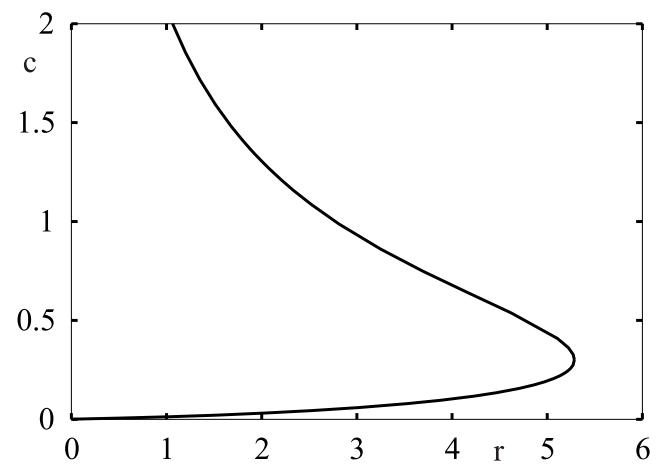

FIG. 3.4. Speed of a traveling pulse as a function of spine stem resistance $r$. Note that propagation failure results for too large a spine-stem resistance. $g_{L}=1.25, h=2.5, \rho=25, \tau_{s}=2$, $\eta_{0}=100$.

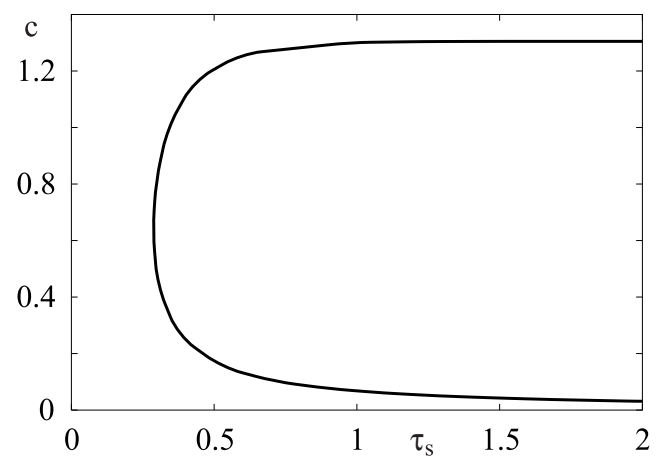

FIG. 3.5. Speed of a traveling pulse as a function of pulse width $\tau_{s}$. Note that for too small a choice of $\tau_{s}$ solitary waves cannot propagate. $g_{L}=1.25, h=2.5, \rho=25, r=2, \eta_{0}=100$.

$(x, t)$ rather than in the moving frame. Since the shape of the traveling pulse is fixed by the function $\eta(t)$, it is natural to consider local perturbations of the firing times given by $T(x)=x / c+u(x)$. A similar approach has recently been applied to the stability of traveling waves in IF systems with synaptic and passive dendritic interactions $[6,7]$. The cable potential $V(x, t)$ satisfying equation $(2.1)$ with the spine potential $\widehat{V}(x, t)=\eta(t-T(x))$ can be evaluated in terms of the Green's function for the infinite cable equation as

$$
V(x, t)=\frac{\rho}{r} \int_{-\infty}^{t} \mathrm{~d} s \int_{-\infty}^{\infty} \mathrm{d} y G(x-y, t-s) \eta(s-T(y))
$$

where

$$
G(x, t)=\frac{1}{\sqrt{4 \pi t}} \mathrm{e}^{-\epsilon t} \mathrm{e}^{-x^{2} / 4 t}
$$

and $G(x, t)$ vanishing for $t<0$. If we now demand that the IF process $(3.4)$ driven by the potential $V(x, t)$ reaches threshold at time $T(x)$ (according to 3.5) 
then we obtain the following self-consistency condition for a traveling pulse (assuming $\left.\lim _{x \rightarrow-\infty} U(x, t)=0\right)$ :

$$
h=\frac{1}{r} \int_{-\infty}^{0} \mathrm{~d} t \mathrm{e}^{\hat{\epsilon} t} V(x, t+T(x))
$$

We now expand equations (4.1) and (4.3) in powers of the perturbation $u(x)$. The zeroth order term generates the self-consistency condition for the speed $c$ of the unperturbed traveling pulse:

$$
h=\frac{1}{r} \int_{-\infty}^{0} \mathrm{~d} t \mathrm{e}^{\hat{\epsilon} t} \bar{V}(x, t+x / c)
$$

where

$$
\bar{V}(x, t)=\frac{\rho}{r} \int_{-\infty}^{t} \mathrm{~d} s \int_{-\infty}^{\infty} \mathrm{d} y G(x-y, t-s) \eta(s-y / c)
$$

We can evaluate equation (4.5) using Fourier transforms. That is, expand $\eta(s)$ as

$$
\eta(s)=\int_{-\infty}^{\infty} \frac{\mathrm{d} k}{2 \pi} \mathrm{e}^{i k s} \widetilde{\eta}(k)
$$

and then perform the integrations over $y$ and $s$ with

$$
\int_{-\infty}^{\infty} \mathrm{d} x \mathrm{e}^{-i k x} G(x, t)=\mathrm{e}^{-\epsilon(k) t}, \quad \epsilon(k)=\epsilon+k^{2}
$$

to obtain

$$
\bar{V}(x, t)=\frac{\rho}{r} \int_{-\infty}^{\infty} \frac{\mathrm{d} k}{2 \pi} \mathrm{e}^{i k(t-x / c)} \frac{\widetilde{\eta}(k)}{\epsilon(k / c)+i k}
$$

Substitution of (4.8) into (4.4) finally gives

$$
h=\frac{\rho}{r^{2}} \int_{-\infty}^{\infty} \frac{\mathrm{d} k}{2 \pi} \frac{\tilde{\eta}(k)}{[\epsilon(k / c)+i k][\hat{\epsilon}+i k]}
$$

When one considers a rectangular pulse-shape for the action potential wave-form of height $\eta_{0}$ and duration $\tau_{s}$ such that

$$
\widetilde{\eta}(k)=\frac{1-\mathrm{e}^{-i k \tau_{s}}}{i k}
$$

then it is a simple matter to check that the dispersion relationship (4.9) reduces to (3.16) derived by alternative means in section 3 .

The first order term in the expansion of equation (4.3) yields a linear equation for the perturbations $u(x)$ from which the linear stability of the traveling pulse can be deduced. This linear equation takes the form

$$
0=\frac{1}{r} \int_{-\infty}^{0} \mathrm{~d} t \mathrm{e}^{\hat{\epsilon} t} \delta_{u} V(x, t)
$$

where

$$
\delta_{u} V(x, t)=\frac{\rho}{r} \int_{-\infty}^{t} \mathrm{~d} s \int_{-\infty}^{\infty} \mathrm{d} y G(x-y, t-s) \eta^{\prime}(s-y / c)[u(x)-u(y)]
$$


Equations (4.11) and (4.12) have solutions of the form $u(x)=\mathrm{e}^{\lambda x}$ with $\lambda$ satisfying the characteristic equation

$$
I(\lambda)=I(0)
$$

where

$$
I(\lambda)=\int_{-\infty}^{\infty} \frac{\mathrm{d} k}{2 \pi} \frac{i k \widetilde{\eta}(k)}{[\epsilon(k / c+i \lambda)+i k][\hat{\epsilon}+i k]}
$$

Asymptotic stability holds if all non-zero solutions of the characteristic equation have negative real part. (The existence of a solution $\lambda=0$ reflects the translation invariance of the underlying dynamical system). Equation (4.14) can be evaluated by closing the contour in the lower-half complex $k$-plane. Since $\eta(s)=0$ for $s<0$ it follows that any poles of $\widetilde{\eta}(k)$ lie in the upper-half complex plane so that we only have to consider poles arising from the zeros of the function $\epsilon(k / c+i \lambda)+i k$. The latter are given explicitly by $k=i k_{ \pm}(\lambda)$ where

$$
\frac{k_{ \pm}(\lambda)}{c}=-\left(\lambda+\frac{c}{2}\right) \pm \sqrt{c^{2} / 4+c \lambda+\epsilon}
$$

Let us decompose $\lambda$ into real and imaginary parts according to $\lambda=\alpha+i \beta$. Then

$$
k_{ \pm}(\lambda)=-u_{ \pm}(\alpha, \beta)-i v_{ \pm}(\alpha, \beta)
$$

with

$$
\frac{u_{ \pm}(\alpha, \beta)}{c}=\alpha+\frac{c}{2} \mp A(\alpha, \beta), \quad \frac{v_{ \pm}(\alpha, \beta)}{c}=\beta \mp B(\alpha, \beta)
$$

and (for $\beta>0$ )

$$
\begin{aligned}
& A(\alpha, \beta)=\sqrt{\frac{1}{2}\left[R(\alpha)+\sqrt{R(\alpha)^{2}+c^{2} \beta^{2}}\right]} \\
& B(\alpha, \beta)=\sqrt{\frac{1}{2}\left[-R(\alpha)+\sqrt{R(\alpha)^{2}+c^{2} \beta^{2}}\right]}
\end{aligned}
$$

where

$$
R(\alpha)=\frac{c^{2}}{4}+c \alpha+\epsilon
$$

In order to check stability of a solitary pulse we need to establish that there are no solutions for which $\alpha>0$. We distinguish two cases according to the sign of $u_{+}(\alpha, \beta)$. Case A: $u_{+}(\alpha, \beta)<0$ which reduces to the condition

$$
c^{2} \beta^{2}>4(\alpha+c / 2)^{2}\left(\alpha^{2}-\epsilon\right)
$$

There is then a single pole at $k=i k_{-}(\lambda)$ and equation (4.14) reduces to $I(\lambda)=I_{-}(\lambda)$ where we define

$$
I_{ \pm}(\lambda)=c^{2} \frac{k_{ \pm}(\lambda) \widetilde{\eta}\left(i k_{ \pm}(\lambda)\right)}{\left[k_{+}(\lambda)-k_{-}(\lambda)\right]\left[\hat{\epsilon}-k_{ \pm}(\lambda)\right]}
$$

Case B: $u_{+}(\alpha, \beta)>0$ and which there are poles at $k=i k_{ \pm}$so that $I(\lambda)=I_{-}(\lambda)-$ $I_{+}(\lambda)$. 
For concreteness let us return to the step-like pulse considered earlier. Substituting equations (4.16) and (4.10) into (4.21) and equating real and imaginary parts yields

$$
\begin{aligned}
& \operatorname{Re} I_{ \pm}(\lambda)=c \frac{X_{ \pm}\left[A\left(\hat{\epsilon}+u_{ \pm}\right)-B v_{ \pm}\right]+Y_{ \pm}\left[B\left(\hat{\epsilon}+u_{ \pm}\right)+A v_{ \pm}\right]}{2\left[A^{2}+B^{2}\right]\left[\left(\hat{\epsilon}+u_{ \pm}\right)^{2}+v_{ \pm}^{2}\right]} \\
& \operatorname{Im} I_{ \pm}(\lambda)=c \frac{Y_{ \pm}\left[A\left(\hat{\epsilon}+u_{ \pm}\right)-B v_{ \pm}\right]-X_{ \pm}\left[B\left(\hat{\epsilon}+u_{ \pm}\right)+A v_{ \pm}\right]}{2\left[A^{2}+B^{2}\right]\left[\left(\hat{\epsilon}+u_{ \pm}\right)^{2}+v_{ \pm}^{2}\right]}
\end{aligned}
$$

where

$$
\begin{aligned}
X_{ \pm} & \equiv \operatorname{Re}\left\{k_{ \pm}(\lambda) \widetilde{\eta}\left(i k_{ \pm}(\lambda)\right)\right\}=\eta_{0}\left[\mathrm{e}^{-u_{ \pm} \tau_{s}} \cos \left(v_{ \pm} \tau_{s}\right)-1\right] \\
Y_{ \pm} & \equiv \operatorname{Im}\left\{k_{ \pm}(\lambda) \widetilde{\eta}\left(i k_{ \pm}(\lambda)\right)\right\}=-\eta_{0} \mathrm{e}^{-u_{ \pm} \tau_{s}} \sin \left(v_{ \pm} \tau_{s}\right)
\end{aligned}
$$

One may then determine the stability of solution branches in the $(c, \rho),(c, r)$ or $\left(c, \tau_{s}\right)$ plane by simultaneously solving $\operatorname{Re} I(\lambda)-\operatorname{Re} I(0)=0$ and $\operatorname{Im} I(\lambda)-\operatorname{Im} I(0)=0$ for $\alpha$ and $\beta$, with $c$ determined by (4.9) (or equivalently (3.16) for the specific case of a rectangular pulse shape for the action potential). A numerical solution of this problem (see figure 4.1) shows that, for example, one may obtain solutions with $\beta=0$ and $\alpha<0$ along the upper branch of figure 3.3. Moreover, $\alpha$ changes sign as it passes through the point where $\mathrm{d} \rho / \mathrm{d} c=0$ in the $(c, \rho)$ plane whilst moving from the upper solution branch to the lower. Hence, of the two possible traveling wave solutions the faster one is stable. This result is consistent with direct numerical evolution of the equations of motion (see figure 3.3). Other solutions with $\alpha<0$ and $\beta>0$ are also found for both the fast and slow branches but do not affect the above stability argument.

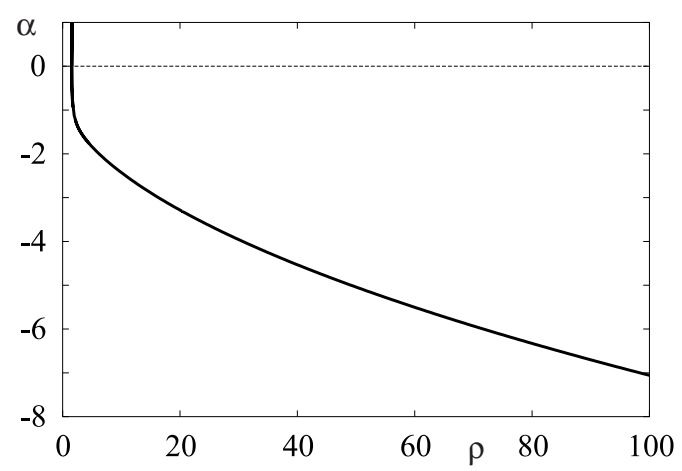

FIG. 4.1. A plot of the eigenvalues arising in the linearization of the IF model shows that solutions with $\alpha<0$ and $\beta=0$ can be found for the branch of solutions with greatest speed. The graph above shows the behavior of $\alpha=\operatorname{Re}(\lambda)$ with $\beta=0$ for the upper solution branch of figure 3.3 Note that $\alpha=0$ at the point in the $(c, \rho)$ plane at which the speed of the two possible traveling waves becomes equal. For the slower branch one finds that there exists solutions in the $(\alpha, \beta)$ plane with $\alpha>0$ indicating that the slower branch is unstable.

5. Passive properties of spines. Till now we have paid little attention to the passive properties of spines. However, this is an important area of study in its own right and has been pursued by many authors $[21,22,39,32,33,15]$. Here we briefly show how one might succinctly study the properties of passive spines in the Baer and 
Rinzel model in the absence of any active membrane. We do this to clarify the role of the passive electrical properties of dendritic spines in spike initiation. For instance, it is possible to show that, for large spine head resistances, the response of the tree to current injected at the spine head may be expressed in terms of the response to current injected directly into the shaft. Dropping the terms describing the currents due to active membrane, ie ignoring terms with voltage dependent gating variables, the Baer and Rinzel system (2.1) and (2.2) becomes linear, with $I(\widehat{V}, m, n, h)=-\widehat{\epsilon} \widehat{V}$, and may be solved by Fourier transforming spatial variables and Laplace transforming time dependent ones. We introduce the following notation for these transforms:

$$
\begin{aligned}
V(k, t) & =\int_{-\infty}^{\infty} \mathrm{d} x \mathrm{e}^{-i k x} V(x, t) \\
V(x, E) & =\int_{0}^{\infty} \mathrm{d} t \mathrm{e}^{-E t} V(x, t)
\end{aligned}
$$

There are two possible ways to inject a current into the system; i) directly onto the shaft so that one must add an injected current term to the right hand side of (2.1) and ii) into the spine head so that one must instead add this term to the right hand side of (2.2). For example, adding a term $J(x, t)$ to the right hand side of $(2.2)$ means that the solution may be written

$$
V(k, E)=\frac{\rho}{r}\left[1-\frac{\rho}{r^{2}} G(k, E) \widehat{G}(E)\right]^{-1} G(k, E) \widehat{G}(E) J(k, E)
$$

where

$$
\begin{aligned}
G(k, E) & =\left(k^{2}+E+\epsilon\right)^{-1} \\
\widehat{G}(E) & =(E+\hat{\epsilon})^{-1}
\end{aligned}
$$

Denoting the response of the cable in case i) as $V(x, t)$ and to case ii) as $V^{*}(x, t)$ it may be shown that

$$
V^{*}(k, E)=\frac{\rho}{r} \widehat{G}(E) V(k, E)
$$

If $\hat{\epsilon} \gg 1$ then it is possible that $\widehat{G}(E)$ is a relatively flat function of $E$ compared to $V(k, E)$. In this case the major contribution of $\widehat{G}(E)$ to $V^{*}(x, t)$ will come from $\widehat{G}(0)$. From biological data it appears that $r g_{L} \ll 1[22]$ so that we may take $\epsilon \gg 1$ assuming $r g_{L} \ll 1$ and $r \ll 1$. In this case $\widehat{G}(0)=r$ so that $V^{*}(x, t)=\rho V(x, t)$. The response of the cable to current injected directly onto the shaft or into the spines connected to the shaft are proportional (by a factor of $\rho$ ). Hence, for the continuum model, it may be easier to initiate a traveling wave by directly stimulating the group of spines above a point on the shaft rather than by directly stimulating a patch of membrane on the shaft itself with an excitatory input. A further discussion of the best ways in which to initiate a chain reaction in a dendritic system with excitable spines can be found in the paper by Baer and Rinzel [3].

6. Discussion. In this paper we used reduction methods to derive an integrateand-fire (IF) model of wave propagation along a dendritic cable with active spines. This allowed us to construct analytical expressions for both the wave speed and wave profile along the cable as a function of various neurophysiological parameters. Moreover, we were able to determine the stability of solutions using the linearized map of 
firing times generated by the IF process in the spine-head. In particular, we found that the speed of a stable wave decreased as a function of both spine density and spine stem resistance. Propagation failure was shown to occur for too low a spine density or too high a spine-stem resistance. In spite of its simplicity, the IF model was able to reproduce behavior consistent with that observed in simulations of more detailed biophysical models.

A potentially important application of our analysis is to the study of how active dendrites contribute to the input-output response properties of a neuron. As an illustrative example, suppose that several distal sites labelled $p=1, \ldots P$ are stimulated at times $s_{i}$ and each event triggers a traveling wave as constructed in section 3 . Assuming that the generated pulses do not interact with each other, the resulting potential seen at the soma would then be of the form $V_{\text {tot }}(t)=\sum_{p=1}^{P} V\left(t-s_{i}-\tau_{i}\right)$ with discrete delays $\tau_{i}=x_{i} / c$ where $x_{i}$ is the electronic distance of the $i$ th stimulated site from the soma and $c$ is the wave speed. Taking the soma itself to evolve according to IF dynamics leads to an effective two-layer IF model of a neuron with active dendrites. Such a model provides a starting point for the investigation of the computational role of active spines in neuronal processing. For example, one might calculate the sensitivity of the firing rate at the soma in response to the exact spatial arrangement of activated spine-heads in the dendritic tree. Such calculations are particularly relevant to the understanding of how complex cells in visual cortex can exhibit orientation tuning [26]. More detailed extensions of the model would need to take into account the branching structure of the tree including non-uniform geometries, interactions between solitary pulses, and the effects of back-propagating action potentials. Indeed one might hope to model the observed clustering of spines with a simple piecewise linear function for the spine density, whilst branching structures are most easily analyzed with the Green's function techniques discussed in [8]. Numerical studies of dendritic cables with uniform density of active spines but with nonuniform dendritic radius have already demonstrated the possibility of propagation failure into the thicker part of the dendrite as well as the possibility of reversal or echo waves [41].

Finally it is worth pointing out the formal similarities of our reduced IF model with that of a recently introduced system for the study of travelling waves observed when $\mathrm{Ca}^{2+}$ is released from internal stores in living cells. In the fire-diffuse-fire (FDF) model of calcium release $[28,16,31]$ an array of point-source $\mathrm{Ca}^{2+}$ release sites is embedded in a continuum in which $\mathrm{Ca}^{2+}$ ions diffuse. To model the effect of $\mathrm{Ca}^{2+}$ induced $\mathrm{Ca}^{2+}$ release (CICR) it is assumed that $\mathrm{Ca}^{2+}$ is released from the array of point sources whenever the concentration of $\mathrm{Ca}^{2+}$ at a release site exceeds some threshold. To mimic the self-regulatory nature of a real $\mathrm{Ca}^{2+}$ puff the release of $\mathrm{Ca}^{2+}$ is modelled as a pulse of a certain strength and duration. Under certain conditions the release of $\mathrm{Ca}^{2+}$ from one site allows diffusion of ions to a neighboring site where a release event may be initiated provided the density of $\mathrm{Ca}^{2+}$ exceeds the threshold for the CICR mechanism. This process is thought to underly the generation of saltatory $\mathrm{Ca}^{2+}$ waves observed in living cells. A correspondence between the FDF model and the model introduced in this paper can be obtained by identifying the continuous media of the FDF model with a passive dendritic cable and the $\mathrm{Ca}^{2+}$ release sites with active spines. However, in the FDF model there is no resetting of the state variable describing the dynamics of the release sites. As a result we view the IF model of dendritic cable with active spines as a spike-diffuse-spike model (SDS). Both FDF and SDS type systems are capable of composing global signals (travelling waves) from elementary events (puffs or spikes). A study of the effect of ion pumps (that 
transport $\mathrm{Ca}^{2+}$ back into the stores) on the speed of travelling waves may be found in [12] where the correspondence between the two models is explored further. Note also that, unlike the FDF model, the SDS model as currently formulated is a continuum model. A lattice version of this model, with active spines at specific sites on a dendritic tree, will be explored elsewhere. 
Appendix A. For the Hodgkin-Huxley model of excitable nerve tissue it is common practice to write

$$
\begin{aligned}
\tau_{X}(\widehat{V}) & =\frac{1}{\alpha_{X}(\widehat{V})+\beta_{X}(\widehat{V})} \\
X_{\infty}(\widehat{V}) & =\alpha_{X}(\widehat{V}) \tau_{X}(\widehat{V})
\end{aligned}
$$

for $X \in\{m, n, h\}$ where

$$
\begin{aligned}
\alpha_{m}(\widehat{V}) & =\frac{0.1(\widehat{V}+40)}{1-\exp [-0.1(\widehat{V}+40)]} \\
\alpha_{h}(\widehat{V}) & =0.07 \exp [-0.05(\widehat{V}+65)] \\
\alpha_{n}(\widehat{V}) & =\frac{0.01(\widehat{V}+55)}{1-\exp [-0.1(\widehat{V}+55)]} \\
\beta_{m}(\widehat{V}) & =4.0 \exp [-0.0556(\widehat{V}+65)] \\
\beta_{h}(\widehat{V}) & =\frac{1}{1+\exp [-0.1(\widehat{V}+35)]} \\
\beta_{n}(\widehat{V}) & =0.125 \exp [-0.0125(\widehat{V}+65)]
\end{aligned}
$$

All potentials are measured in $\mathrm{mV}$, all times in ms and all currents in $\mu \mathrm{A}$ per $\mathrm{cm}^{2}$. In obtaining a reduction of the full Hodgkin-Huxley equations we use the following parameter values: $\widehat{C}=1 \mu \mathrm{F} \mathrm{cm}{ }^{-2}, g_{L}=0.3 \mathrm{mmho} \mathrm{cm}^{-2}, g_{K}=36 \mathrm{mmho} \mathrm{cm}^{-2}$, $g_{N a}=120 \mathrm{mmho} \mathrm{cm}^{-2}, V_{L}=-54.402 \mathrm{mV}, V_{K}=-77 \mathrm{mV}$ and $V_{N a}=50 \mathrm{mV}$.

\section{REFERENCES}

[1] L. F. Аввотт And K. I. Blum, Functional significance of long-term potentiation for sequence learning and prediction, Cerebral Cortex, 6 (1996), pp. 406-416.

[2] L. F. Aвbott And T. B. Kepler, Model neurons: from Hodgkin-Huxley to Hopfield, in Statistical Mechanics of Neural Networks, L. Garrido, ed., no. 368 in Lecture notes in Physics, Springer-Verlag, 1990, pp. 5-18.

[3] S. M. BAER AND J. Rinzel, Propagation of dendritic spines mediated by excitable spines: A continuum theory, Journal of Neurophysiology, 65 (1991), pp. 874-890.

[4] S. M. BAER AND C. TIER, An analysis of a dendritic neuron model with an active membrane site, Journal of Mathematical Biology, 23 (1986), pp. 137-161.

[5] W. J. BEYN, The numerical computation of connecting orbits in dynamical systems, IMA Journal of Numerical Analysis, 9 (1990), pp. 379-405.

[6] P. C. Bressloff, Synaptically generated wave propagation in excitable neural media, Physical Review Letters, 82 (1999), pp. 2979-2982.

[7] - Traveling waves and pulses in a one-dimensional network of excitable integrate-and-fire neurons, Journal of Mathematical Biology (to appear), (1999).

[8] P. C. Bressloff and S. Coombes, Physics of the extended neuron, International Journal of Modern Physics B, 11 (1997), pp. 2343-2392.

[9] - A dynamical theory of spike train transitions in networks of integrate-and-fire oscillators, SIAM Journal on Applied Mathematics (to appear), (1999).

[10] P. Chen ANd J. Bell, Spine-density dependence of the qualitative behaviour of a model of a nerve fiber with excitable spines, Journal of Mathematical Analysis and Applications, 187 (1994), pp. 384-410.

[11] W. R. Chen, J. Midtganrd, And G. M. Shepherd, Forward and backward propagation of dendritic impulses and their synaptic control in mitral cells, Science, 278 (1997), pp. 463467.

[12] S. CoOmbes, The effect of ion pumps on the speed of travelling waves in the fire-diffuse-fire model of $\mathrm{Ca}^{2+}$ release, Proceedings of the Royal Society A (submitted), (1999). 
[13] B. ERmentrout, The analysis of synaptically generated travelling waves, Journal of Computational Neuroscience, 5 (1998), pp. 191-208.

[14] D. Johnston, J. C. Magee, C. M. Colbert, And B. R. Christie, Active properties of neuronal dendrites, Annual Review of Neuroscience, 19 (1996), pp. 165-186.

[15] M. Kawato And N. Tsukahara, Electrical properties of dendritic spines with bulbous end terminals, Biophysical Journal, 46 (1984), pp. 155-166.

[16] J. E. Keizer, G. D. Smith, S. Ponce-Dawson, And J. Pearson, Saltatory propagation of $C a^{2+}$ waves by $\mathrm{Ca}^{2+}$ sparks, Biophysical Journal, 75 (1998), pp. 595-600.

[17] T. B. Kepler, L. F. AbBott, And E. Marder, Reduction of conductance-based neuron models, Biological Cybernetics, 66 (1992), pp. 381-387.

[18] W. M. Kistler, W. Gerstner, and J. L. van Hemmen, Reduction of the Hodgkin-Huxley equations to a single-variable threshold, Neural Computation, 9 (1997), pp. 1015-1045.

[19] C. Koch, Methods in Neuronal Modeling: From Synapses to Networks, MIT Press, 1989.

[20] - Biophysics of Computation, Oxford University Press, 1999.

[21] C. Koch And T. Poggio, Electrical properties of dendritic spines, Trends in Neuroscience, (1983), pp. 80-83.

$[22]-$, A theoretical analysis of electrical properties of dendritic spines, Proceedings of the Royal Society London B, 218 (1983), pp. 455-477.

[23] M. E. Larkum, J. J. Zhu, and B. Sakmann, A new cellular mechanism for coupling inputs arriving at different cortical layers, Nature, 398 (1999), pp. 338-341.

[24] M. V. Mascagni And A. S. Sherman, Numerical methods for neuronal modelling, in Methods in Neuronal Modeling : From Ions to Networks, C. Kock and I. Segev, eds., MIT Press, 1998.

[25] B. W. MEL, NMDA-based pattern discrimination in a modeled cortical neuron, Neural Computation, 4 (1992), pp. 502-517.

[26] - Why have dendrites? a computational perspective, in Dendrites, G. Stuart, N. Spruston, and M. Hausser, eds., Oxford University Press, 1999.

[27] J. P. Miller, W. RAll, AND J. Rinzel, Synaptic amplification by active membrane in dendritic spines, Brain Research, 325 (1985), pp. 325-330.

[28] J. E. Pearson and S. Ponce-Dawson, Crisis on skid row, Physica A, 257 (1998), pp. 141-148.

[29] D. H. Perkel and D. J. Perkel, Dendritic spines: role of active membrane in modulating synaptic efficacy, Brain Research, 325 (1985), pp. 331-335.

[30] R. S. Petersen, Dendritic spines: the backbone of single neuron computation, Master's thesis, King's College London, 1992.

[31] S. Ponce-Dawson, J. Keizer, and J. E. Pearson, Fire-diffuse-fire model of dynamics of intracellular Calcium waves, Proceedings of the National Academy of Sciences USA, 96 (1999), pp. 6060-6063.

[32] F. Pongracz, The function of dendritic spines, Neuroscience, 15 (1985), pp. 933-946.

[33] F. Pongracz, J. Martos, and F. Zsuppan, Nerve cells with irregular processes: Demonstration of anisotropic core geometry of a pyramidal cell, Neuroscience, 25 (1988), pp. 10771094.

[34] J. RinzeL, Excitation dynamics: insights from simplified membrane models, Federation Proceedings, 44 (1985), pp. 2944-2946.

[35] I. Segev And W. Rall, Computational study of an excitable dendritic spine, Journal of Neurophysiology, 60 (1988), pp. 499-523.

[36] — Excitable dendrites and spines: earlier theoretical insights elucidate recent direct observations, Trends in Neuroscience, 21 (1998), pp. 453-460.

[37] G. M. Shepherd AND R. K. BRAYTON, Logic operations are properties of computer-simulated interactions between excitable dendritic spines, Neuroscience, 21 (1987), pp. 151-165.

[38] G. M. Shepherd, R. K. Brayton, J. P. Miller, I. Segev, J. Rinzel, and W. Rall, Signal enhancement in distal cortical dendrites by means of interactions between active dendritic spines, Proceedings of the National Academy of Sciences USA, 82 (1985), pp. 2192-2195.

[39] C. J. Wilson, Passive cable properties of dendritic spines and spiny neurons, The Journal of Neuroscience, 4 (1984), pp. 281-297.

[40] Y. ZHOU, Unique wave front for dendritic spines with Nagumo dynamics, Mathematical Biosciences, 148 (1998), pp. 205-225.

[41] Y. Zhou AND J. Bell, Study of propagation along nonuniform excitable fibers, Mathematical Biosciences, 119 (1994), pp. 169-203. 